\title{
Variant histology in bladder cancer: diagnostic and clinical implications
}

\author{
Anna J. Black, Peter C. Black \\ Department of Urologic Sciences, University of British Columbia, Vancouver, Canada \\ Contributions: (I) Conception and design: PC Black; (II) Administrative support: None; (III) Provision of study materials or patients: None; (IV) \\ Collection and assembly of data: AJ Black; (V) Data analysis and interpretation: AJ Black; (VI) Manuscript writing: All authors; (VII) Final approval \\ of manuscript: All authors. \\ Correspondence to: Peter C. Black, MD, FACS, FRCSC. Level 1, 2775 Laurel St., Vancouver BC, V5Z 1M9, Canada. Email: pblack@mail.ubc.ca.
}

\begin{abstract}
Urothelial carcinoma differentiates into variant histological subtypes in approximately $25 \%$ of cases. Since every histological variant has unique characteristics, including metastatic potential, expression of immunotherapy targets, and susceptibility to radiation or chemotherapy, every variant offers a unique diagnostic and therapeutic challenge. However, since any single variant is relatively rare, there is a risk of missed pathological diagnosis and sub-optimal clinical management. Ensuring awareness among pathologists and wide-spread familiarity with the nuances of variants among urologists is therefore essential. Additionally, variant histologies may act as an intermediate between classical clinicopathological staging of bladder cancer and evolving molecular classification. Therefore, this review aims to provide a brief overview of the diagnostic, prognostic, and therapeutic implications of each variant histologic subtype. Despite the development of standardized diagnostic criteria, the diagnosis of variant histologies continues to pose a challenge and results in significant interobserver variability. The prognosis of any single variant is poorly studied. However, squamous and glandular differentiation are thought to have little effect on prognosis while micropapillary, sarcomatoid, plasmacytoid, and small cell carcinomas are associated with a poor prognosis. Although evidence surrounding therapeutic strategies is sparse, management guidelines have been developed for variant histologies, which are often treated more aggressively than pure urothelial carcinoma. For example, the presence of variant histology warrants radical cystectomy in patients with T1 disease. Recommendations surrounding neo-adjuvant chemotherapy and radiation therapy also differ with each variant. As new treatments emerge for advanced bladder cancer, studying outcomes in each variant will become critical. Since prognosis and management hinge on the presence of variant histologies, accurate diagnosis and a thorough understanding of these variants are imperative for optimal management of urothelial carcinoma.
\end{abstract}

Keywords: Bladder cancer; variant histology; diagnosis; treatment; prognosis

Submitted May 24, 2020. Accepted for publication Jun 11, 2020.

doi: $10.21037 /$ tcr-20-2169

View this article at: http://dx.doi.org/10.21037/tcr-20-2169

\section{Introduction}

The propensity of urothelial carcinoma of the bladder to differentiate along divergent histologic paths has been recognized for many years. Approximately $25 \%$ of bladder tumors harbor variant histology, which can offer unique diagnostic and therapeutic challenges that are critical to the optimal management of bladder cancer overall $(1,2)$.
Although any single variant may be considered relatively rare, together they make up a significant subset of patients. It is therefore essential that the practicing urologist be facile with the evolving nuances of these different entities. Furthermore, in an era of evolving molecularly targeted therapy, variant histology is a bridge between classic clinicopathologic characterization of bladder cancer and the 


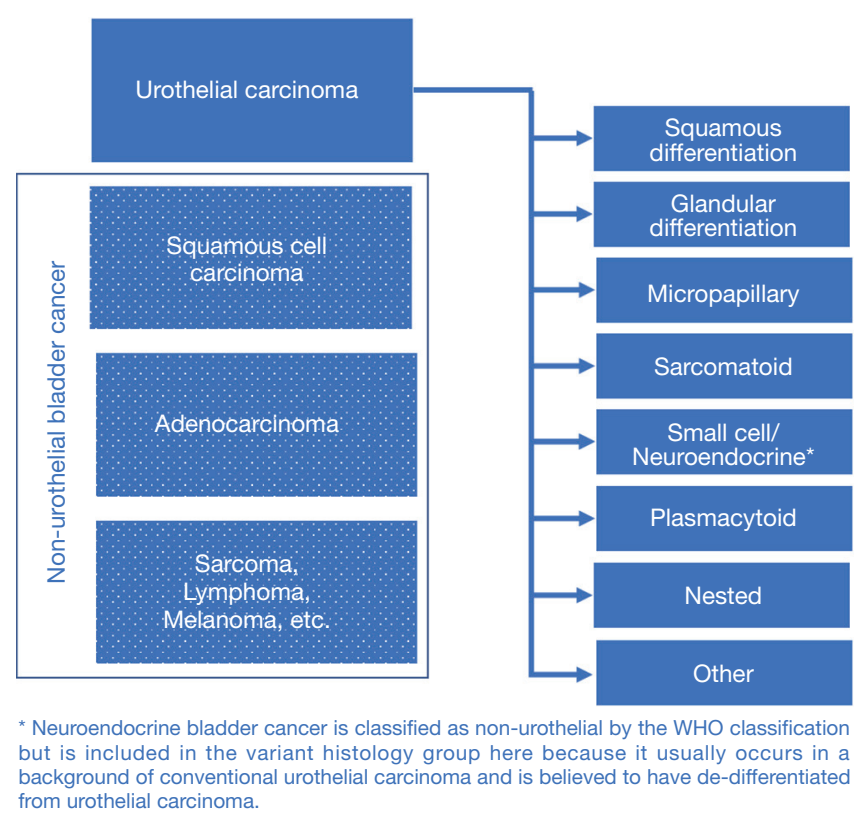

Figure 1 The World Health Organization (WHO) 2016 classification of urothelial tract tumors.

advancing molecular classification of bladder cancer.

The histologic classification of bladder cancer follows the World Health Organization (WHO) classification of tumors of the urinary tract $(2,3)$. The language used to describe conventional bladder cancer has evolved from the more traditional term "transitional cell carcinoma" to the now more commonly used term "urothelial carcinoma" $(2,3)$. "Transitional cell" describes the morphology of the urothelium, but transitional cells can also be found in other anatomic locations, so that "urothelial" is more specific to the genitourinary tract. Urothelial carcinoma with variant histology describes any morphologic variant of conventional urothelial carcinoma that is believed to be derived from urothelial carcinoma and is typically admixed with conventional urothelial carcinoma in the same tumor. This can be delineated from non-urothelial carcinomas, such as squamous cell carcinoma and adenocarcinoma, which also grow in the bladder but are not derived from urothelial carcinoma and have a distinct biology (Figure 1). Neuroendocrine tumors of the bladder represent a gray area. They are classified as non-urothelial even though they are widely believed to result from de-differentiation of urothelial carcinoma and are molecularly similar to urothelial carcinoma $(4,5)$. Neuroendocrine bladder cancer has therefore been included as a variant in this review.
The objective of this review is to highlight the important diagnostic, prognostic and therapeutic implications of the most commonly encountered variant histologies in bladder cancer. For a more comprehensive review the reader is referred to the recent Société Internationale d'Urologie (SIU) International Consultation on Urological Diseases (ICUD) review of bladder cancer pathology (6).

\section{Diagnostic implications}

Variant histology is so common in bladder cancer that it is critical for the pathologist always to be thinking of these variants when evaluating histologic sections of a bladder tumor. Awareness of the different variants is the first challenge, especially for the pathologist not focused on genitourinary pathology, who is more likely to miss a variant (7). There is a high interobserver variability in the assessment of histologic variants, so that even expert genitourinary pathologists will frequently provide disparate reports (8). Moreover, despite attempts to develop standardized criteria, much subjectivity remains in the determination of histologic variants $(2,8)$. Additional challenges for the pathologist include the fact that most variants are present only in a minority of any given tumor, and there is often a discrepancy between the histology from the transurethral resection and from radical cystectomy.

Terminology for variant histology in bladder cancer is also critical, especially for the clinician not focused on bladder cancer. Education of the urologist is essential, since the terms used to describe variant histologies maybe either under-recognized or misinterpreted. A term such as "micropapillary" may be misconstrued to be relatively benign since "papillary" itself is a favorable feature in bladder cancer. "Plasmacytoid" is not a new term, but it has replaced "signet ring" carcinoma in the conventional terminology used by urologists and pathologists, and its grave impact on prognosis should not be overlooked. "Signet ring" is also used in the context of mucin-producing adenocarcinoma, especially in the gastrointestinal tract, but urothelial plasmacytoid carcinoma lacks extracellular mucin and represents a distinct entity. The terms "small cell" and "neuroendocrine" carcinoma are used interchangeably in bladder cancer, which in many cases is justified. However, "neuroendocrine" generally refers to a broader classification that includes large cell neuroendocrine bladder cancer and some benign entities such as paraganglioma. Large cell neuroendocrine bladder cancer is much rarer and is managed like small cell neuroendocrine carcinoma due to 
the absence of evidence suggesting it should be managed differently.

\section{Squamous and glandular differentiation}

Squamous differentiation can be prominent enough that it is difficult to distinguish this entity from true squamous cell carcinoma. When there is any doubt, retained expression of GATA-3 or uroplakin can help confirm the diagnosis of urothelial carcinoma with squamous differentiation (9). Glandular differentiation, on the other hand, is less common and is less likely to be mistaken for primary or metastatic adenocarcinoma.

\section{Micropapillary carcinoma}

Micropapillary carcinoma has garnered the most attention of the variant histologies over the past several years, and may therefore be more familiar to many pathologists than some other variants. Micropapillary bladder cancer is characterized by tight clusters of high-grade tumor cells that lack a fibrovascular core and are surrounded by retraction spaces $(10,11)$. However, there is no consensus on strict criteria for determining micropapillary carcinoma, and the subtleties of borderline cases make detection challenging.

\section{Sarcomatoid}

Sarcomatoid bladder cancer is characterized by a histologic appearance that resembles an intermediate between a true epithelial carcinoma and a sarcoma. It expresses hallmark epithelial markers, but at the same time demonstrates highgrade spindle cell morphology. Sarcomatoid urothelial carcinoma expresses mesenchymal markers such as ZEB1 and TWIST1 that indicate that it has undergone epithelial-to-mesenchymal transition, which is a potential pathogenic mechanism of sarcomatoid differentiation (12). Carcinosarcoma is a separate entity on the spectrum between urothelial carcinoma and true sarcoma (13). It represents an admixture of urothelial carcinoma with true sarcomatous elements. Both sarcomatoid carcinoma and carcinosarcoma must be distinguished from true sarcoma.

\section{Small cell/neuroendocrine}

Neuroendocrine tumors can occur in different tissues, including especially the gastrointestinal tract where they originate from cells that have both a nerve-like appearance and also contain secretory granules like endocrine cells. Neuroendocrine tumors can be either well differentiated (e.g., paraganglioma) or poorly differentiated, and the latter can be either small cell or large cell. When the morphology is not clear, immunohistochemistry for classic neuroendocrine markers such as chromogranin and synaptophysin is used for confirmation of the diagnosis.

\section{Plasmacytoid}

Plasmacytoid urothelial carcinoma represents a particularly aggressive histologic variant (14). This is the subtype, for example, that can cause a linitis plastica-like diffuse infiltration of the bladder wall and it is often associated with peritoneal carcinomatosis. The name plasmacytoid is derived from the appearance of the cells in the tumor that resemble the $\mathrm{B}$ cells of the immune system. The abundant cytoplasm and eccentric nuclei in plasmacytoid carcinoma have given rise to the alternative name "signet ring" carcinoma for this variant. One common underlying molecular feature of plasmacytoid urothelial carcinoma, which was observed in $84 \%$ of cases in one series, is the loss of E-cadherin expression by immunohistochemistry which is explained by a truncating mutation or by promoter hypermethylation of the $C D H 1$ gene (15). E-cadherin is a cell adhesion molecule that also serves as a prototypical marker of epithelial differentiation. It is therefore logical that loss of E-cadherin leads to the discohesive, invasive, and metastatic plasmacytoid carcinoma.

\section{Nested variant}

The nested variant of bladder cancer is noteworthy due to its relatively benign appearance. It is recognized by characteristic small solid nests of benign appearing neoplastic cells that can be difficult to differentiate from von Brunn's nests. The key with the nested variant is to recognize its malignant potential (16). There is no evidence that it behaves clinically any different than conventional urothelial carcinoma and there is no evidence to adjust treatment based on this histology.

\section{Future paradigms in diagnosis of bladder cancer variants}

Two advances could impact the classification of bladder cancer beyond histologic variants. Firstly, molecular classification could offer insight into the biological differences reflected in the aberrant histomorphology. 
Molecular patterns could also be used to delineate a more refined classification of disease. Building on the RNAbased molecular classification of urothelial carcinoma, the most prominent example of the convergence of histology and molecular characterization lies in the identification of a neuroendocrine-like subtype of bladder cancer. This subtype maintains a conventional urothelial appearance but has a neuroendocrine gene expression and a very poor prognosis consistent with true neuroendocrine bladder cancer (17-19).

Molecular evaluations have shown clear differences between the histologic subtypes, although this has not been used for disease classification. E-cadherin loss in plasmacytoid carcinoma, for example, has already been discussed. Micropapillary carcinoma similarly has frequent HER2/ErbB2 amplification, but this is observed also in conventional urothelial carcinoma and may not be useful for classification $(20,21)$. Gene expression analysis has revealed that micropapillary carcinoma develops along a luminal pathway and is characterized by the activation of miR-296 and RUVBL1 target genes (22).

The other major advance that could improve differentiation between histologic variants is the clinical adoption of artificial intelligence (AI). A recent paper demonstrates that AI can be used to distinguish the molecular subtypes of bladder cancer (12). AI is dependent on a large sample size and it will require cooperation of the bladder cancer research community to assemble enough cases with variant histology to be able to use this technology moving forward.

\section{Prognostic implications}

Many studies have assessed the prognostic importance of variant histology, and globally variant histology can be considered an adverse risk feature $(23,24)$. Since tumors with variant histology often present with higher stage, it is essential to assess prognosis after correcting for stage (25). Unfortunately, clinical staging of bladder cancer is poor and the published results are therefore often disparate.

The relative impact of variant histology on clinical outcomes needs to be considered in specific disease states. Most of the literature has focused on the non-metastatic muscle invasive setting, especially after radical cystectomy. Without much evidence there is also some consensus that variant histology portends a poor prognosis in the nonmuscle invasive T1 setting. In the metastatic setting there is less consensus and less evidence about impact on treatment and outcomes. Clinical trials for locally advanced and metastatic urothelial carcinoma typically exclude patients with more than $50 \%$ variant histology.

Squamous and glandular differentiation appear to have little impact on prognosis. Some series report inferior outcomes while others report equal outcomes to conventional urothelial carcinoma (26-28). Some of this variability is likely related to the threshold for detection of squamous differentiation which varies widely in the literature. The other variants, including especially micropapillary, sarcomatoid, plasmacytoid and small cell carcinoma are associated with higher stage disease, a higher risk of recurrence and progression, and decreased cancer specific and overall survival (29).

\section{Treatment implications}

It remains unclear whether the proportion of tumor that is involved with the variant histology has real clinical impact, and whether treatment should be based on the proportion of variant histology. The consensus has evolved that any portion of variant histology should lead the tumor to be treated as if it were that variant. The one exception is perhaps in the metastatic and locally advanced setting where patients are often excluded from trials only if more than $50 \%$ of the tumor represents variant histology.

Although the evidence is sometimes lacking, there are clear guidelines on the management of variant histologies in bladder cancer. A recent consensus statement was published from an international collaboration of 113 bladder cancer experts under the auspices of the European Association of Urology-European Society of Medical Oncology Guidelines Committees (30). Nonetheless, some controversies also remain. Squamous differentiation, glandular differentiation, and nested variant do not require any modifications to management compared to conventional urothelial carcinoma. Implications of the other subtypes for treatment will be discussed in the following according to disease state.

\section{Non-muscle invasive bladder cancer}

With the general belief that variant histology is an adverse pathologic feature that increases the risk of understaging and is associated with poor disease outcomes, there is consensus amongst bladder cancer experts that any T1 tumor with variant histology should be considered for immediate cystectomy if the patient is fit for cystectomy. There is little evidence about the efficacy 


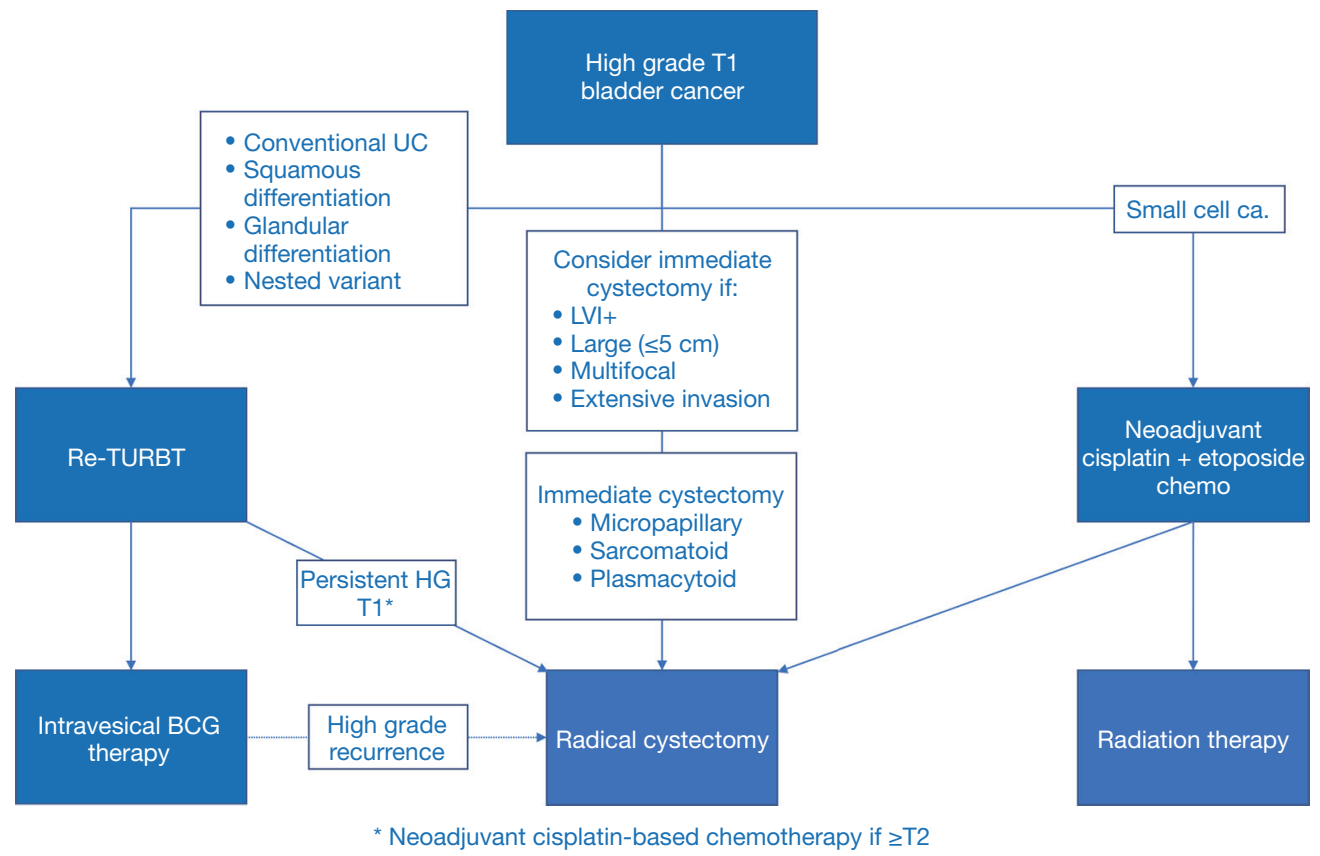

Figure 2 Management of non-muscle invasive bladder cancer with variant histology. TURBT, transurethral resection of bladder tumor; BCG, Bacillus Calmette-Guerin.

of Bacillus Calmette-Guerin (BCG) in this setting and the recommendation is really based on a conservative approach to minimize the risk to the patient. If the patient is not fit for cystectomy or declines cystectomy, an aggressive repeat transurethral resection of bladder tumor (TURBT) is recommended but the value of BCG is unknown.

The best evidence for immediate cystectomy for $\mathrm{T} 1$ variant histology has been reported in the context of micropapillary urothelial carcinoma. One single institutional series of 66 patients demonstrated that patients had better 5 -year disease-specific survival with immediate cystectomy (100\%) compared to treatment with BCG followed by possible salvage cystectomy (60\%) (31). On the other hand, in another series of 36 patients, the disease-specific survival at 5 years was $75 \%$ in BCG-treated patients and $83 \%$ after radical cystectomy $(\mathrm{P}=0.9)$ (32). An analysis of 430,000 bladder cancer patients in the National Cancer Database identified 869 patients with micropapillary bladder cancer and compared outcomes of those treated with bladderpreserving surgery or radical cystectomy (33). There was no survival difference between the 2 cohorts. Although the evidence is weak, a survey of urologists indicated that most recommend immediate cystectomy in patients with micropapillary T1 bladder cancer (31).

T1 small cell carcinoma represents a unique entity, as systemic chemotherapy followed by radical cystectomy or radiotherapy is the suggested standard therapy. The management of T1 bladder cancer with variant histology is summarized in Figure 2.

\section{Muscle invasive bladder cancer}

Variant histology could potentially impact two key clinical decisions in the management of muscle invasive bladder cancer: (I) consideration of radiotherapy as a standard alternative to radical cystectomy, and (II) the use of neoadjuvant chemotherapy.

Due to a paucity of evidence, radical cystectomy is often preferred to radiotherapy for muscle invasive variant histology other than squamous/glandular differentiation. In the Massachusetts General Hospital experience, only 16 of 303 patients treated with trimodal therapy had variant histology other than squamous/glandular differentiation, and variant histology did not correlate with outcome (34). In principle, we would expect squamous and glandular components to be radiation-sensitive. Furthermore, small cell carcinoma is known to be highly radiosensitive, and small cell carcinoma of the lung is routinely treated with radiation. There should be equipoise around the role of radical cystectomy versus radiation therapy after 
chemotherapy for localized small cell carcinoma $(35,36)$. The predominant risk of recurrence is systemic and both treatments are likely to provide similar local control. However, we know nothing about differential effects of surgery versus radiation for micropapillary, plasmacytoid, or sarcomatoid urothelial carcinoma.

Although it is clear that variant histology leads to an adverse prognosis and an increased risk of upstaging, which would imply that these patients warrant treatment intensification with neoadjuvant chemotherapy, it is not clear that cisplatin-based chemotherapy is effective against variant histology and there is therefore a risk of disease progression during potentially futile neoadjuvant chemotherapy which could negatively impact outcomes (37).

Squamous or glandular differentiation may be a favorable risk feature in patients receiving neoadjuvant chemotherapy prior to radical cystectomy. In the landmark S8710 trial, squamous and/or glandular differentiation was associated with a worse prognosis compared to conventional urothelial carcinoma in patients undergoing radical cystectomy alone, but with a better prognosis in patients treated with cisplatin-based chemotherapy before cystectomy (38). This is consistent with the observation that basal-like tumors, according to the RNA-based molecular classification, also have a poor outcome without neoadjuvant chemotherapy but perform as well as the most favorable subtype when treated with chemotherapy (39). Since primary squamous cell carcinoma is considered less responsive to chemotherapy, it is important to highlight that bladder cancer with squamous differentiation may be more responsive.

There are three published series looking at the impact of neoadjuvant chemotherapy in patients with muscle invasive micropapillary bladder cancer. In 154 patients from the National Cancer Database, neoadjuvant chemotherapy was administered in 35 , and 119 underwent immediate cystectomy (40). The risk of extravesical extension was reduced from $73 \%$ to $46 \%$ but there was no overall survival benefit. The 5 -year overall survival was $63 \%$ which compared to $71 \%$ in patients not receiving neoadjuvant chemotherapy. In the second series of 40 patients with muscle invasive micropapillary urothelial carcinoma who received neoadjuvant chemotherapy, 15 (45\%) were downstaged to pT0, but there was no apparent overall survival benefit (41). Finally, in the third series, neoadjuvant chemotherapy appeared to improve survival in patients with muscle invasive micropapillary bladder cancer only in the absence of concomitant hydronephrosis (42).
None of these series are large enough to anticipate that a survival advantage would be expected with neoadjuvant chemotherapy, especially in a retrospective analysis with likely selection bias, but they do confirm that the pathologic response of micropapillary urothelial carcinoma to neoadjuvant chemotherapy appears to be similar to conventional urothelial carcinoma.

Treatment algorithms for small cell carcinoma of the bladder borrow from evidence-based algorithms for small cell lung cancer $(36,43)$. Chemotherapy is the mainstay of treatment in lung because small cell carcinoma metastasizes early but is highly chemosensitive. The paradigm for small cell bladder cancer is therefore primary chemotherapy followed by consolidative local therapy. The optimal chemotherapy regimen is thought to be cisplatin/etoposide because that is the standard in small cell lung cancer.

Small cell carcinoma is noteworthy for early metastasis and a propensity to spread to bones and brain. As results, staging should include a CT or MRI of the head and a bone scan. There is not yet compelling evidence to administer prophylactic brain radiation, as there is for small cell carcinoma of the lung. In one series from MD Anderson Cancer Center $50 \%$ of patients with T3-T4, node positive, or metastatic bladder cancer had brain metastases (43). There is therefore a trial underway at this center testing prophylactic cranial irradiation in patients with $\geq \mathrm{cT} 3 \mathrm{~b}$ disease.

The single arm PURE-01 trial testing systemic administration of the immune checkpoint inhibitor pembrolizumab prior to radical cystectomy for muscle invasive bladder cancer included also patients with variant histology, and therefore provides an early glimpse into the relative benefit of immunotherapy in variant histology compared to conventional urothelial carcinoma (44). Thirty-four of 114 patients in this trial had a component of variant histology, and 19 had $>50 \%$ variant histology. A broad spectrum of variants was represented. Downstaging to $\leq$ ypT1N0 was observed in $56 \%$ of pure urothelial carcinoma, $67 \%$ of variant histology if it represented $\leq 50 \%$ of the tumor, and $42 \%$ of variant histology if it represented $>50 \%$ of the tumor. However, responses were only observed in patients with squamous differentiation or lymphoepithelioma-like variant, and there were no responses in any of the remaining nine patients with other variants. More studies are required to explore the role of immunotherapy in variant histologies.

The management of muscle invasive bladder cancer with variant histology is summarized in Figure 3. 


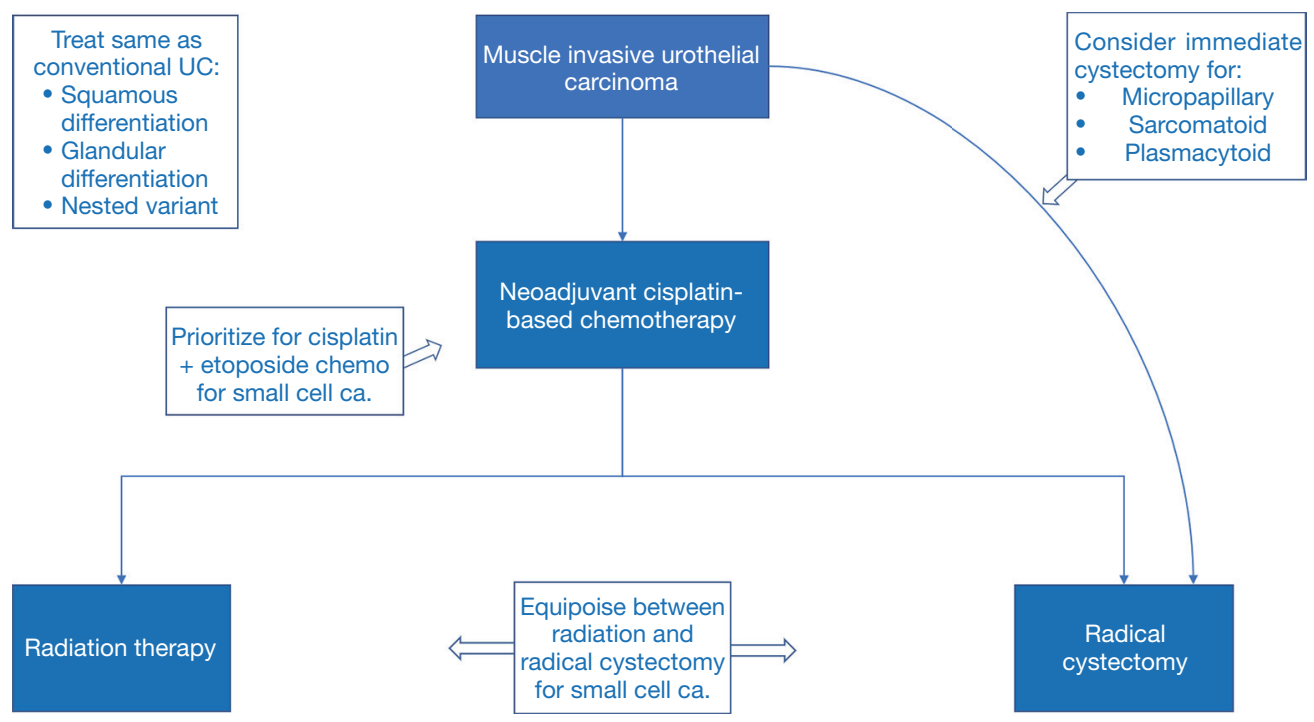

Figure 3 Management of non-metastatic muscle invasive bladder cancer with variant histology.

\section{Metastatic bladder cancer}

Variant histology has up until now had few direct implications for management of patients with metastatic urothelial carcinoma. Conventional cisplatin or carboplatinbased chemotherapy is administered to these patients and second-line therapies including immunotherapy, fibroblast growth factor receptor (FGFR)-targeted therapy and enfortumab vedotin do not differentiate by histologic variants $(45,46)$. There is an absence of evidence regarding the efficacy of all of these agents in patients with variant histology, and they are therefore treated similarly to conventional urothelial carcinoma.

One study of PD-L1 expression according to histologic subtype found higher expression in sarcomatoid carcinoma compared to conventional urothelial carcinoma, but otherwise similar expression across subtypes (47). However, PD-L1 expression has not correlated well with response to immunotherapy in urothelial carcinoma.

The major trials of immune checkpoint inhibitors in the post-platinum metastatic and locally advanced setting included patients with $\leq 50 \%$ variant histology, but the results for this subgroup have not usually been reported specifically. The Keynote-045 trial comparing pembrolizumab to chemotherapy in this disease state reported on subgroup analysis that patients with mixed histology $(\mathrm{n}=155)$ had greater relative overall survival benefit with pembrolizumab [hazard ratio (HR) 0.58 ] than patients with urothelial carcinoma ( $\mathrm{n}=383$; HR 0.80) (48).
A single arm phase II trial testing nivolumab (antiPD-1) and ipilimumab (anti-CTLA4) in advanced rare genitourinary cancers included five patients with urothelial carcinoma of the bladder containing variant histology, in addition to a larger subset of 14 patients with primary adenocarcinoma or squamous cell carcinoma. In a preliminary report two of three small cell cases had an objective response, while the sole plasmacytoid tumor also responded and the sole sarcomatoid tumor did not (49).

In a multicenter retrospective analysis of outcomes after immune checkpoint blockade (mostly atezolizumab and pembrolizumab) in 395 patients with advanced urothelial carcinoma, the objective response rate was $28 \%$ for conventional urothelial carcinoma $(70 \%$ of patients) and $29 \%$ for mixed variant and conventional urothelial carcinoma (30\% of patients) (50). The study does not describe what proportion of patients were platinum-refractory versus first line cisplatin-ineligible. The response rate was somewhat higher in sarcomatoid carcinoma [38\% in 16 patients; odds ratio (OR) 1.51 (95\% confidence interval (CI): 0.53-4.30)] and somewhat lower in plasmacytoid carcinoma ( $17 \%$ in 12 patients; OR 0.50 (95\% CI: 0.11-2.35)), but otherwise similar in squamous differentiation (28\%), micropapillary (28\%), and glandular differentiation (20\%). The proportion of variant histology was not available to these authors. The progression-free and overall survival was similar between conventional urothelial carcinoma and variant histology. The sample size for each cohort is too small to draw any definitive conclusions, but 
importantly there is no indication that any one subtype should not receive immunotherapy.

There were also eight patients with neuroendocrine urothelial carcinoma in this multicenter study, and 2 (25\%) responded to therapy, but only for a median of 13 weeks. The progression-free and overall survival of this small subgroup was markedly worse than the rest of the variant histology group and the conventional urothelial carcinoma group. This puts into question a prior report from Kim et al. indicating a $100 \%$ objective response rate to atezolizumab in 11 patients with platinum-refractory metastatic or locally advanced urothelial carcinoma who had a neuroendocrinelike gene expression pattern despite conventional urothelial histology. Although immunotherapy plus platinum/ etoposide has become first-line therapy for extensive stage small cell lung cancer, there is no indication that small cell carcinoma is particularly responsive to immunotherapy (51).

In the pivotal BLC2001 trial testing the FGFR inhibitor erdafitinib in platinum-ineligible and platinum-refractory patients, patients were excluded if the tumor contained $\geq 50 \%$ variant histology. The report of the trial results does not include mention of variant histology. However, since enrolment in this trial depended on the presence of an FGFR2 or FGFR3 alteration, one would anticipate that only few variants would be represented in the study population, although the mutational profiles of most variants have been poorly studied. Micropapillary tumors are nearly all luminal, and FGFR3 alterations are most frequent in luminal tumors, but FGFR3 alterations rarely coincide with ErbB2 alterations, which are common in micropapillary carcinoma (22).

The pivotal EV-210 trial testing enfortumab vedotin in the third line after platinum chemotherapy and immunotherapy included patients with variant histology without restrictions ( $12 \%$ squamous differentiation and $21 \%$ other variant histology), but did not report outcomes in this subgroup (46). However, another group demonstrated that the expression of Nectin 4 , the target of enfortumab, is highly dependent on histologic subtype (52). All 8 pure small cell tumors and the small cell component of all 7 mixed tumors lacked Nectin 4 expression. Similarly, all 7 carcinosarcomas lacked Nectin 4 expression.

HER2 is over-expressed in up to $75 \%$ of micropapillary urothelial carcinoma and the corresponding gene ErbB2 is amplified in approximately one third of cases. Although it has not yet been tested, this finding suggests that micropapillary urothelial carcinoma could be particularly amenable to HER2-targeted therapy (53). While HER2- targeted therapy has yet to show efficacy in bladder cancer, an antibody drug conjugate that uses the HER2 expression simply as a target for delivery of a cytotoxic payload remains a promising paradigm.

\section{Conclusions}

A familiarity with histological variants of urothelial carcinoma is crucial for both pathologists and clinicians to facilitate appropriate diagnosis and management of patients. While approximately $25 \%$ of urothelial carcinomas harbor variant histology, each individual variant is relatively rare. Therefore, pathologists not specialized in bladder cancer may easily miss variants, while urologists may underrecognize or misinterpret the terms used to describe variant histologies. Although standardized criteria have been developed to address diagnostic accuracy of urothelial carcinoma, interobserver variability in the diagnosis of histological variants remains high.

The rarity of each histological variant also means that prognosis and optimal management are understudied. However, while squamous and glandular differentiation likely have little effect on prognosis, micropapillary, sarcomatoid, plasmacytoid, and small cell carcinoma are all associated with poorer outcomes than pure urothelial carcinoma. The metastatic potential, expression of immunotherapy targets, and susceptibility to radiation or chemotherapy differ with variant histology, thus therapeutic strategies also differ. Although there are clear guidelines on the management of variant histologies, these are largely based on subgroup analyses, relatively small studies, expert consensus, or are even applications of treatments used in similar cancers in other organ sites (e.g., small cell lung cancer). Therefore, both an awareness of the current evidence surrounding variant histologies and further research are crucial to optimize the treatment of urothelial carcinoma.

\section{Acknowledgments}

Funding: None.

\section{Footnote}

Provenance and Peer Review: This article was commissioned by the Guest Editors (Dr. Ja Hyeon Ku, Dr. Hyeong Dong Yuk, and Dr. Hyung Suk Kim) for the series "Urothelial Carcinoma" published in Translational Cancer Research. The 
article was sent for external peer review organized by the Guest Editors and the editorial office.

Conflicts of Interest: Both authors have completed the ICMJE uniform disclosure form (available at http:// dx.doi.org/10.21037/tcr-20-2169). The series "Urothelial Carcinoma" was commissioned by the editorial office without any funding or sponsorship. The authors have no conflicts of interest to declare.

Ethical Statement: The authors are accountable for all aspects of the work in ensuring that questions related to the accuracy or integrity of any part of the work are appropriately investigated and resolved.

Open Access Statement: This is an Open Access article distributed in accordance with the Creative Commons Attribution-NonCommercial-NoDerivs 4.0 International License (CC BY-NC-ND 4.0), which permits the noncommercial replication and distribution of the article with the strict proviso that no changes or edits are made and the original work is properly cited (including links to both the formal publication through the relevant DOI and the license). See: https://creativecommons.org/licenses/by-nc-nd/4.0/.

\section{References}

1. Ploeg M, Aben KK, de Kaa C, et al. Clinical Epidemiology of Nonurothelial Bladder Cancer: Analysis of The Netherlands Cancer Registry. J Urol 2010;183:915-20.

2. Humphrey PA, Moch H, Cubilla AL, et al. The 2016 WHO Classification of Tumours of the Urinary System and Male Genital Organs_-Part B: Prostate and Bladder Tumours. Eur Urol 2016;70:106-19.

3. Epstein JI, Amin MB, Reuter VR, et al. The World Health Organization/ International Society of Urological Pathology Consensus Classification of Urothelial (Transitional Cell) Neoplasms of the Urinary Bladder. Am J Surg Pathol 1998;22:1435-48.

4. Shen P, Jing Y, Zhang R, et al. Comprehensive genomic profiling of neuroendocrine bladder cancer pinpoints molecular origin and potential therapeutics. Oncogene 2018;37:3039-44.

5. Wang L, Smith BA, Balanis NG, et al. A genetically defined disease model reveals that urothelial cells can initiate divergent bladder cancer phenotypes. Proc Natl Acad Sci U S A 2020;117:563-72.

6. Compérat, EM. Chapter 2, Pathology. In: Black P,
Gontero P. editors. Bladder Cancer. A Joint SIU-ICUD International Consultation. Lisbon, Portugal. October 19, 2017:83-175. Available online: http://www.siu-urology. org/society/siu-icud

7. Shah RB, Montgomery JS, Montie JE, et al. Variant (divergent) histologic differentiation in urothelial carcinoma is under-recognized in community practice: impact of mandatory central pathology review at a large referral hospital. Urol Oncol 2013;31:1650-5.

8. Luchey AM, Manimala NJ, Dickinson S, et al. Change in Management Based on Pathologic Second Opinion Among Bladder Cancer Patients Presenting to a Comprehensive Cancer Center: Implications for Clinical Practice. Urology 2016;93:130-4.

9. Gulmann C, Paner GP, Parakh RS, et al. Immunohistochemical profile to distinguish urothelial from squamous differentiation in carcinomas of urothelial tract. Hum Pathol 2013;44:164-72.

10. Amin MB, Ro JY, el-Sharkawy T, et al. Micropapillary variant of transitional cell carcinoma of the urinary bladder. Histologic pattern resembling ovarian papillary serous carcinoma. Am J Surg Pathol 1994;18:1224-32.

11. Kiss B, Wyatt AW, Douglas J, et al. Her2 alterations in muscle-invasive bladder cancer: Patient selection beyond protein expression for targeted therapy. Sci Rep 2017;7:42713.

12. Genitsch V, Kollár A, Vandekerkhove G, et al. Morphologic and genomic characterization of urothelial to sarcomatoid transition in muscle-invasive bladder cancer. Urol Oncol 2019;37:826-36.

13. Lopez-Beltran A, Pacelli A, Rothenberg HJ, et al. Carcinosarcoma and sarcomatoid carcinoma of the bladder: clinicopathological study of 41 cases. J Urol 1998;159:1497-503.

14. Perrino CM, Eble J, Kao CS, et al. Plasmacytoid/ diffuse urothelial carcinoma: a single-institution immunohistochemical and molecular study of 69 patients. Hum Pathol 2019;90:27-36.

15. Al-Ahmadie HA, Iyer G, Lee BH, et al. Frequent somatic CDH1 loss-of-function mutations in plasmacytoid variant bladder cancer. Nat Genet 2016;48:356-8.

16. Zhong M, Tian W, Zhuge J, et al. Distinguishing Nested Variants of Urothelial Carcinoma From Benign Mimickers by TERT Promoter Mutation. Am J Surg Pathol 2015;39:127-31.

17. Kamoun A, de Reyniès A, Allory Y, et al. A Consensus Molecular Classification of Muscle-invasive Bladder Cancer. Eur Urol 2020;77:420-33. 
18. Batista da Costa J, Gibb EA, Bivalacqua TJ, et al. Molecular Characterization of Neuroendocrine-like Bladder Cancer. Clin Cancer Res 2019;25:3908-20.

19. Grivas P, Bismar TA, Alva AS, et al. Validation of a neuroendocrine-like classifier confirms poor outcomes in patients with bladder cancer treated with cisplatin-based neoadjuvant chemotherapy. Urol Oncol 2020;38:262-8.

20. Ching CB, Amin MB, Tubbs RR, et al. HER2 gene amplification occurs frequently in the micropapillary variant of urothelial carcinoma: analysis by dual-color in situ hybridization. Mod Pathol 2011;24:1111-9.

21. Ross JS, Wang K, Gay LM, et al. A High Frequency of Activating Extracellular Domain ERBB2 (HER2) Mutation in Micropapillary Urothelial Carcinoma. Clin Cancer Res 2014;20:68-75.

22. Guo CC, Dadhania V, Zhang L, et al. Gene Expression Profile of the Clinically Aggressive Micropapillary Variant of Bladder Cancer. Eur Urol 2016;70:611-20.

23. Black PC, Brown GA, Dinney CP. The impact of variant histology on the outcome of bladder cancer treated with curative intent. Urol Oncol 2009;27:3-7.

24. Veskimäe E, Espinos EL, Bruins HM, et al. What Is the Prognostic and Clinical Importance of Urothelial and Nonurothelial Histological Variants of Bladder Cancer in Predicting Oncological Outcomes in Patients with Muscleinvasive and Metastatic Bladder Cancer? A European Association of Urology Muscle Invasive and Metastatic Bladder Cancer Guidelines Panel Systematic Review. Eur Urol Oncol 2019;2:625-42.

25. Li Q, Assel M, Benfante NE, et al. The Impact of Plasmacytoid Variant Histology on the Survival of Patients with Urothelial Carcinoma of Bladder after Radical Cystectomy. Eur Urol Focus 2019;5:104-8.

26. Ehdaie B, Maschino A, Shariat SF, et al. Comparative Outcomes of Pure Squamous Cell Carcinoma and Urothelial Carcinoma With Squamous Differentiation in Patients Treated With Radical Cystectomy. J Urol 2012;187:74-9.

27. Kim SP, Frank I, Cheville JC, et al. The Impact of Squamous and Glandular Differentiation on Survival After Radical Cystectomy for Urothelial Carcinoma. J Urol 2012;188:405-9.

28. Lin X, Deng T, Wu S, et al. The clinicopathological characteristics and prognostic value of squamous differentiation in patients with bladder urothelial carcinoma: a meta-analysis. World J Urol 2020;38:323-33.

29. Wright JL, Black PC, Brown GA, et al. Differences in Survival Among Patients With Sarcomatoid Carcinoma,
Carcinosarcoma and Urothelial Carcinoma of the Bladder. J Urol 2007;178:2302-6; discussion 2307.

30. Witjes JA, Babjuk M, Bellmunt J, et al. EAU-ESMO Consensus Statements on the Management of Advanced and Variant Bladder Cancer-An International Collaborative Multistakeholder Effort: Under the Auspices of the EAU-ESMO Guidelines Committees. Eur Urol 2020;77:223-50.

31. Willis DL, Flaig TW, Hansel DE, et al. Micropapillary bladder cancer: Current treatment patterns and review of the literature. Urol Oncol 2014;32:826-32.

32. Spaliviero M, Dalbagni G, Bochner BH, et al. Clinical Outcome of Patients with T1 Micropapillary Urothelial Carcinoma of the Bladder. J Urol 2014;192:702-7.

33. Sui W, Matulay JT, James MB, et al. Micropapillary Bladder Cancer: Insights from the National Cancer Database. Bladder Cancer 2016;2:415-23.

34. Krasnow RE, Drumm M, Roberts HJ, et al. Clinical Outcomes of Patients with Histologic Variants of Urothelial Cancer Treated with Trimodality Bladdersparing Therapy. Eur Urol 2017;72:54-60.

35. Patel SG, Stimson CJ, Zaid HB, et al. Locoregional Small Cell Carcinoma of the Bladder: Clinical Characteristics and Treatment Patterns. J Urol 2014;191:329-34.

36. Lynch SP, Shen Y, Kamat A, et al. Neoadjuvant Chemotherapy in Small Cell Urothelial Cancer Improves Pathologic Downstaging and Long-term Outcomes: Results from a Retrospective Study at the MD Anderson Cancer Center. Eur Urol 2013;64:307-13.

37. Culp SH, Dickstein RJ, Grossman HB, et al. Refining Patient Selection for Neoadjuvant Chemotherapy before Radical Cystectomy. J Urol 2014;191:40-7.

38. Scosyrev E, Ely BW, Messing EM, et al. Do mixed histological features affect survival benefit from neoadjuvant platinum-based combination chemotherapy in patients with locally advanced bladder cancer? A secondary analysis of Southwest Oncology Group-Directed Intergroup Study (S8710). BJU Int 2011;108:693-9.

39. Seiler R, Ashab HAD, Erho N, et al. Impact of Molecular Subtypes in Muscle-invasive Bladder Cancer on Predicting Response and Survival after Neoadjuvant Chemotherapy. Eur Urol 2017;72:544-54.

40. Vetterlein MW, Mullane S, Seisen T, et al. Neoadjuvant chemotherapy prior to radical cystectomy for muscleinvasive bladder cancer with variant histology. Cancer 2017;123:4346-55.

41. Meeks JJ, Taylor JM, Matsushita K, et al. Pathological response to neoadjuvant chemotherapy for muscle-invasive 
micropapillary bladder cancer. BJU Int 2013;111:E325-30.

42. Fernández MI, Williams SB, Willis DL, et al. Clinical risk stratification in patients with surgically resectable micropapillary bladder cancer. BJU Int 2017;119:684-91.

43. Siefker-Radtke AO, Kamat AM, Grossman HB, et al. Phase II Clinical Trial of Neoadjuvant Alternating Doublet Chemotherapy With Ifosfamide/Doxorubicin and Etoposide/Cisplatin in Small-Cell Urothelial Cancer. J Clin Oncol 2009;27:2592-7.

44. Necchi A, Raggi D, Gallina A, et al. Updated Results of PURE-01 with Preliminary Activity of Neoadjuvant Pembrolizumab in Patients with Muscle-invasive Bladder Carcinoma with Variant Histologies. Eur Urol 2020;77:439-46.

45. Royce TJ, Lin CC, Gray PJ, et al. Clinical characteristics and outcomes of nonurothelial cell carcinoma of the bladder: Results from the National Cancer Data Base. Urol Oncol 2018;36:78.e1-78.e12.

46. Rosenberg JE, O’Donnell PH, Balar AV, et al. Pivotal Trial of Enfortumab Vedotin in Urothelial Carcinoma After Platinum and Anti-Programmed Death 1/Programmed Death Ligand 1 Therapy. J Clin Oncol 2019;37:2592-600.

47. Li H, Zhang Q, Shuman L, et al. Evaluation of PD-L1 and other immune markers in bladder urothelial carcinoma stratified by histologic variants and molecular subtypes. Sci Rep 2020;10:1439.

48. Bellmunt J, de Wit R, Vaughn DJ, et al. Pembrolizumab as Second-Line Therapy for Advanced Urothelial Carcinoma. N Engl J Med 2017;376:1015-26.

49. McGregor BA, Campbell MT, Xie W, et al. Phase II study of nivolumab and ipilimumab for advanced bladder cancer of variant histologies (BCVH). J Clin Oncol 2019;37:abstr 4518.

50. Miller NJ, Khaki AR, Diamantopoulos LN, et al. Histological Subtypes and Response to PD-1/PD-L1 Blockade in Advanced Urothelial Cancer: A Retrospective Study. J Urol 2020;204:63-70.

51. Horn L, Mansfield AS, Szcz sna A, et al. First-Line Atezolizumab plus Chemotherapy in Extensive-Stage Small-Cell Lung Cancer. N Engl J Med 2018;379:2220-9.

52. Hoffman-Censits JH, Choi W, Lombardo K, et al. Expression of nectin-4 in bladder cancer with variant histology. J Clin Oncol 2020;38:546.

53. Zinnall U, Weyerer V, Compérat E, et al. Micropapillary urothelial carcinoma: evaluation of HER2 status and immunohistochemical characterization of the molecular subtype. Hum Pathol 2018;80:55-64.
Cite this article as: Black AJ, Black PC. Variant histology in bladder cancer: diagnostic and clinical implications. Transl Cancer Res 2020;9(10):6565-6575. doi: 10.21037/tcr-20-2169 